\title{
Calculation of phase transitions of uranium dioxide using structure factor in molecular dynamics
}

\author{
Nagornov Yuri", Katz Andrey \\ Togliatti State University, Togliatti, Samara region, Russia
}

Email address:

Nagornov.Yuri@gmail.com (Yu. Nagornov),elfsage@mail.ru (A. Katz)

\section{To cite this article:}

Nagornov Yuri, Katz Andrey. Calculation of Phase Transitions of Uranium Dioxide Using Structure Factor in Molecular Dynamics. International Journal of Materials Science and Applications. Vol. 2, No. 6, 2013, pp. 228-232. doi: 10.11648/j.ijmsa.20130206.19

\begin{abstract}
We calculate phase transitions of uranium dioxide using structure factor in molecular dynamics. Our method is based on analysis of the rate of the structure factor change with the temperature. The temperatures of melting and transition to the superionic state for the uranium dioxide obtained by this method are $3100 \mathrm{~K}$ and $2600 \mathrm{~K}$, respectively. Theses temperatures much better conform to the experimental values of $3120 \mathrm{~K}$ and $2670 \mathrm{~K}$ than in the radial distribution function analysis method. Other methods give the melting temperatures substantially higher $(3435-3600 \mathrm{~K})$ than the experimental value.
\end{abstract}

Keywords: Uranium Dioxide, Calculation of Phase Transitions, Rate of the Structure Factor Change, Molecular Dynamics

\section{Introduction}

At present, the molecular dynamics (MD) method is widely employed for the simulation of thermodynamic and nonequilibrium processes $[1,2]$. One application of the MD method in material science is to determine the phase transition temperatures. There are various approaches for the MD simulation of the phase transition. One of them is the use of two atom regions separated by the interface, where one region corresponds to the molten state, while the other is associated with the crystal phase. Then, the MD calculation is performed at the given temperature. Some time after, the thermodynamic equilibrium is established, and the system behavior is determined. If the interface shifts towards more intense melting, this means the energy of atoms in the crystal phase area is enough for melting, and vice versa. The paper Ref. [2] uses this approach to determine phase transition temperature of stoichiometric uranium dioxide as $3400-3600 \mathrm{~K}$, which is by $300-500 \mathrm{~K}$ higher than the experimental value (3120K).

Another method is based on the analysis of a radial distribution function of particle number density. Maximums of this function correspond to coordination spheres in a crystal. As the temperature grows, the peak amplitude gradually decreases, and melting causes them to disorder. In particular, the number of the peaks and the distance between them change, which denotes the atom system disordering. However, this approach gives overrated values for the melting point since the MD calculation time does not exceed few nanoseconds. The melting process cannot be accomplished during such a short period, therefore, the MD method is considered to give the overestimated phase transition temperature.

The melting process correlated with the disordering of the structure of atoms in the lattice, which can be calculated when considering diffraction by a grating at different temperatures. It is known [3] that the intensity of the reflection X-ray diffraction or reflection of slow neutrons by the square of the scattering amplitude, which is equal to:

$$
\begin{gathered}
A(\vec{k})=\sum_{m n p} \sum_{i} f_{i} e^{-i\left(\vec{r}_{i}+\vec{r}_{m n p}\right) \vec{k}}= \\
\left(\sum_{m n p} e^{-i \vec{r}_{m n p} \vec{k}}\right)\left(\sum_{i} f_{i} e^{-i \vec{r}_{i} \vec{k}}\right)
\end{gathered}
$$

where $m, n, p$ - index indicating the cell of the crystal, $\vec{r}_{m n p}$ - vector defining the coordinate of the crystal cell, $i$ - the index that defines the atom inside the cell, $\vec{r}_{i}$ - vector defining the coordinates of the atoms relative to the cell, $f_{i}$ - atomic scattering factor, $\vec{k}$ - reciprocal lattice vector.

Considering that the first factor is not equal to zero only in the case when the scattering vector $\vec{k}$ equals to the reciprocal lattice vector $\vec{G}$, we obtain the expression [3]:

$$
A(\vec{k})=\operatorname{MS}(\vec{k})
$$

where $M$ - number of lattice sites, and $S(\vec{k})$ - structural 
factor, equal to:

$$
S(\vec{k})=\sum_{i} f_{i} e^{-i \vec{r}_{i} \vec{k}}
$$

By definition, the intensity of the reflection is equal to the square of the scattering amplitude:

$$
I(\vec{k}) \sim|A(\vec{k})|^{2} \sim|S(\vec{k})|^{2}
$$

It is known that the logarithm of the intensity of the diffraction peaks is proportional to the Debye-Waller factor and decreases almost linearly with temperature [3]. Hence logarithm of the square of the structure factor will decrease linearly with temperature up to the phase transition point, while the conditions of scattering. At point of phase transition the character of the scattering varies, vibrations of lattice atoms increases, and the structure factor decreases sharply. That is why, the article does not focus on the value of the structure factor, but we discuss its dependence on temperature in order to determine the phase transition point from the deflection from linearity.

\section{Calculation Algorithm}

In the classical molecular dynamics method the form and the type of the interatomic potential are postulated, and its parameters are constant regardless of the purpose of calculation. We perform reconstruction of the potential parameters using experimental data of the thermal expansion of the $U O_{2}$ lattice and by enthalpy variation $[1,2]$. We took the most popular rigid ion model that takes into account Coulomb interaction and the short-range Buckingham potential:

$$
U\left(r_{i j}\right)=\frac{z_{i} z_{j}}{r_{i j}}+f \cdot\left(b_{i}+b_{j}\right) \cdot \exp \left(\frac{a_{i}+a_{j}-r_{i j}}{b_{i}+b_{j}}\right)-\frac{c_{i} c_{j}}{r_{i j}{ }^{6}}
$$

Here, the fractional charge value is specified for ions of oxygen and equal 1.2. The charge for uranium ion is obtained by multiplying the value by two (2.4). The parameter $f$ is equal 0.0345 . The other potential parameters were taken from Ref. [4] and are listed in Table 1.

Table 1. Parameters of the potential represented as (5).

\begin{tabular}{c|ccc}
\hline & $\boldsymbol{a}, \AA$ & $\boldsymbol{b}, \AA$ & $\boldsymbol{c}, \mathrm{eV}^{1 / 2} \cdot \AA^{3}$ \\
\hline $\boldsymbol{i}, \boldsymbol{j}=\boldsymbol{U}$ & 1.318 & 0.036 & 0 \\
$\boldsymbol{j}, \boldsymbol{i}=\boldsymbol{O}$ & 1.847 & 0.166 & 4.166 \\
\hline
\end{tabular}

The calculation was carried out on a supercomputer based on two Intel Xeon processors with 160 Gflops of computational power each, and four graphic processors Nvidia Tesla K10 with a peak computational power of 4.58 Tflops each. The software used for simulation was the
DL_POLY 4.04 system developed in Daresbury Laboratory (UK). Source codes of the program were obtained from the developer and were not modified. The system compiled with OpenMPI and CUDA 4.0 parallel processing technologies gives the edge by several orders in computation time over sequential implementation. This hardware-software appliance allowed to perform computations for simulation periods of $10 \mathrm{~ns}$ and more. The potential was set as a file with coefficients from the Table 1 and calculated using (5).

The periodic boundary conditions were applied. The translated cell was selected as a cubic fluorite structure crystal containing from 768 to 12000 ions. All computations implied the integration step of $2 \mathrm{fs}$ and the cut-off radius of $10 \AA$. The Coulomb interactions were treated with the classical Ewald summation technique. Depending on the task, computations were performed for a microcanonical $N V E$ ensemble or a canonical NPT ensemble, but both cases fulfilled isothermal conditions.

Errors were calculated in accordance with root-mean-square deviation, and for measuring the lattice parameter dependence on the temperature they were $0.016-0.018 \%$, and for measuring enthalpy they didn't exceed $0.01 \%$.

\section{Determination of Phase Transition Temperatures}

The interesting way to determine the phase transition temperature is a method in which the structure factor is chosen as the ordering criterion in a crystal [2]. To achieve this goal, the normalized squared modulus of the structure factor is calculated individually for the uranium and oxygen sublattices for (002), (020) and (200) directions. The temperature changes of the structure factor are associated with the changes in the radial distribution function $(R D F)$ of the particle number density. However, the structure factor is more suitable due to its simplicity for comparing the ordering with its numerical estimate. At the temperature of $0 \mathrm{~K}$ the crystal has an ideal structure with the structure factor of one. At high temperatures, on the contrary, for the molten state the squared modulus of the structure factor tends to zero, and its numerical values vary within the range of 0.01-0.04. Unfortunately, similarly to the above mentioned ways, the simulation period is too short to transform the crystal from the solid into the molten state.

Fig. 1 shows the logarithmic dependence of the squared modulus of the structure factor averaged over the three independent directions (002), (020) and (200). The averaging aims to decrease the amplitude of point spread and to simplify the analysis of the results. It is evident that this dependence for oxygen and uranium atoms is linear up to the temperature of $2200 \mathrm{~K}$. 


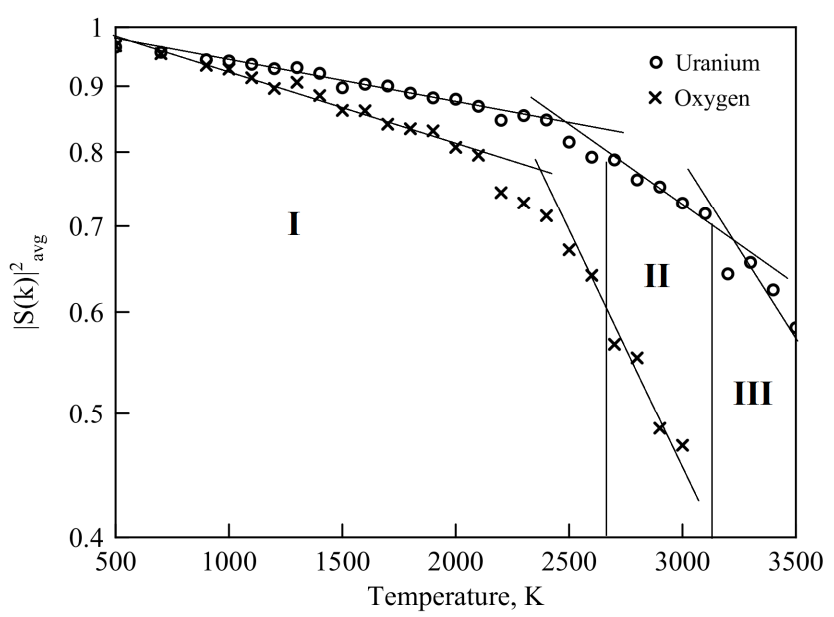

Figure 1. Temperature dependences of squared modulus averaged by directions (002), (020) and (200), separately for uranium and oxygen lattices with linear approximation between phase transition points at $2600 \mathrm{~K}$ and $3100 \mathrm{~K}$. Three areas are shown in figure: I - solid area, II superionic area, III - liquid area. The vertical lines show the two phase transition temperature $-2670 \mathrm{~K}$ and $3120 \mathrm{~K}$.

Fig.1 shows three areas: solid area, superionic area and liquid area, which separated by vertical lines of phase transitions $-2670 \mathrm{~K}$ and $3120 \mathrm{~K}$ corresponding experimental data, as recommended in [11]. In the temperature region $2200-2600 \mathrm{~K}$ dependence is a transitional area, then there is also a linear (region II) to the melting temperature of the lattice uranium $(3120 \mathrm{~K})$.

Note that the transition area of $2200-2600 \mathrm{~K}$, in our opinion, is related with the premelting of oxygen sublattice, since exactly in this temperature range, there are defects $[5,6]$. Calculations imply that the criterion of a defect or a Frenkel pair is the fact of transition of an atom to the interstice space by diffusional processes at simulation temperature. A defect is the atom that resides closer to the center of the interstice than to the node of the lattice. That is, if the parameter of the cubic lattice of a crystal is equal to $a$, the defect threshold is equal to $\sqrt{2} / 4 a$ for the $2 \mathrm{D}$ case (Fig.2) and $\sqrt{3} / 4 a$ for the $3 \mathrm{D}$ case. The radial distribution function changes only due to peaks widening. This fact signifies that oxygen atoms at the temperature of $2250 \mathrm{~K}$ have sufficient kinetic energy and move with high amplitude, which allows them to leave a lattice node and enter the interstice space (Fig.3). Since the modeling time does not exceed $10 \mathrm{~ns}$, the localization of oxygen atoms near the lattice node stays the same.

As seen on Fig. 3 the number of defects in the oxygen sublattice sharply increases, which in turn produces the change in the oscillation amplitude of uranium atoms, and the slope of dependence curves significantly changes. Recent studies have shown $[5,6]$, that the melting process starts at temperatures below the phase transition, the so-called premelting, which is accompanied by the growth of the number of defects.

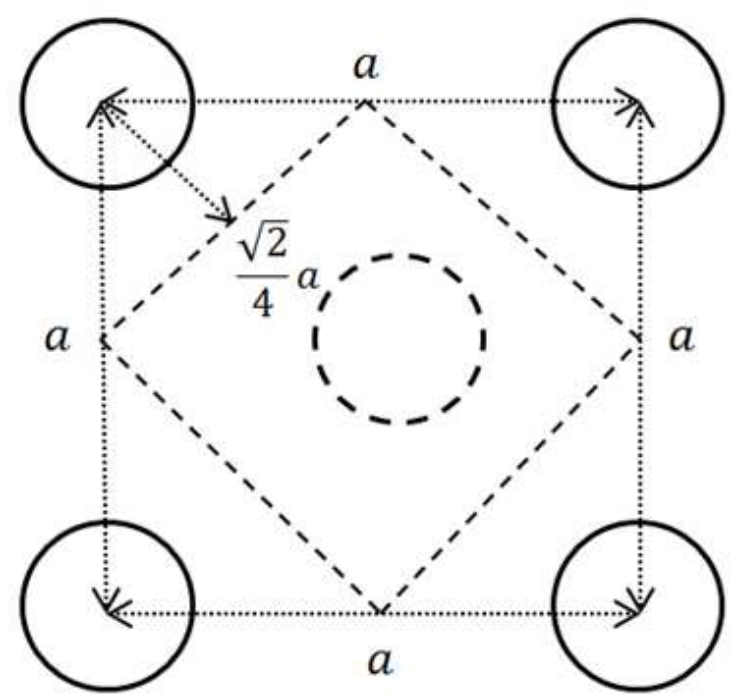

Figure 2. Criteria for determination of number of defects in the oxygen sublattice for $2 D$ case: the inner region is the creation of the defect.

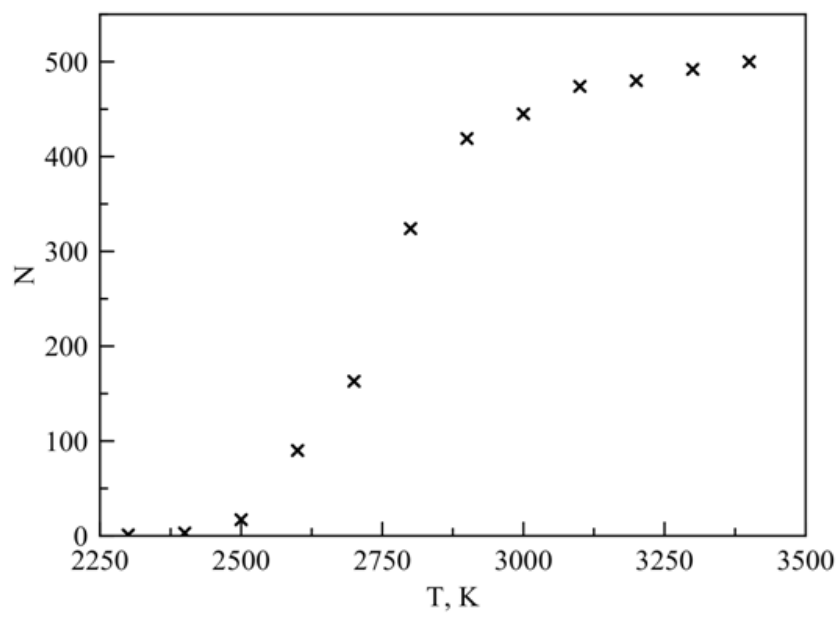

Figure 3. The temperature dependence of the number of defects in the oxygen in $4 \times 4 \times 4$ cell sublattice or 768 molecules of $\mathrm{UO}_{2}$.

Moreover the melting begins at the crystal surface and before reaching melting point the condition are occurred for thermodynamic phase equilibrium between the thin (a few nanometers) liquid film and solid of bulk crystal [7].

In accordance with these views the melting front extends from surface to crystal volume. At each temperature below the melting temperature two phases are coexisted: crystalline and liquid which is thin nanometric layer on the surface. Therefore considered to be the phase transition point is the highest temperature in the transition region (Fig.1, the area between I and II). In our case, this value becomes $2600 \mathrm{~K}$ for oxygen sublattice, and $3100 \mathrm{~K}$ - for uranium sublattice.

Therefore, during the modeling time we can only observe the beginning of melting while the lattice structure stays unaffected. This leads to significant discrepancy of calculated and experimental melting temperature data if only the $R D F$ function is taken into account. On the contrary, selecting as a criterion of the phase transition the 
point where the linear character of the logarithmic dependence of the structural factor on temperature (Fig. 1) changes allows us to determine phase transitions in uranium dioxide crystals with decent accuracy.

\section{Comparison with Other Methods and Potentials}

Papers [5, 6] use the molecular dynamics method to obtain melting temperatures of argon well-matching the experimental data by adding defects and voids to the crystal. The melting temperature is obtained from the transition temperature versus void size curve. We conducted calculations for the ideal crystal and for a crystal with voids produced as a result of exclusion of one molecule of $\mathrm{UO}_{2}$, and the concentration of voids proved to be dependant on the number of translated cells (Fig.4). For uranium dioxide concentration of voids up to $10^{19} \mathrm{~cm}^{-3}$ leaves radial distribution function and, therefore, the melting temperature unaffected. Phase transition to the superionic state happens are the temperature of $2670 \mathrm{~K}$, however, for $100 \mathrm{ps}$ and $10 \mathrm{~ns}$ simulation periods and temperature of $2700 \mathrm{~K}$ there's no difference between the ideal crystal and a crystal with voids. It is clear that as the size of voids increases, the melting temperature decrease, and the reason for that is both concentration of defects and the increase of surface energy. Thus the method of determining the transition temperatures by $R D F$ was not usable in molecular dynamics simulation.

For comparison the melting temperature has been computed in [2] for a few rigid ion potentials [8-10]: Basak, Karakasidis, Morelon, Walker and Yamada. Authors in [2] used two atom regions separated by the interface at initial temperature (Fig.5), where one region corresponds to the molten state, while the other is associated with the crystal phase. When the MD calculation is performed and the thermodynamic equilibrium is established, then the system behavior is determined at final temperature. If the interface boundary shifts towards more intense melting, this means the energy of atoms in the crystal phase area is enough for melting, and vice versa. At temperature melting and the coexistence of liquid and solid UO2 can become energetically possible.

Authors in [2,8-10] proceeded by varying the initial temperature of the simulations and looking (by visualisation of the atoms trajectory) which state is reached by the system after 200 ps. Results of calculations are reported in Table 2 the range of variation of the smoothed curve of temperature, once it became visually stable. These predictions have to be compared to the experimental value of $3120 \pm 20 \mathrm{~K}$ for $\mathrm{UO}_{2}$ [11]. For some runs (Karakasidis potential and Walker potential with an initial temperature of $3200 \mathrm{~K}$ ) it was not clear whether an equilibrium was reached at the end of the simulation or not [2]. It is possible that a longer simulation would end with a solidification of the box. It is seen that the best values obtained for the melting temperature (3435-3600 $\mathrm{K})$ is significantly higher experimental value.

Authors in [2] carried out test which concerns the sensitivity of the predicted temperature to the size of the system. They did not expect to obtain different predictions of the melting temperature, but rather to observe a different behaviour of the interface. Indeed for the smallest systems the ratio of the energy of the separation surface per atom can be quite high and the system will naturally tend to favour the growth of one of the phases. For larger systems, the interfacial energy is relatively less important to the total energy of the system and the coexistence of liquid and solid $\mathrm{UO}_{2}$ can become energetically possible. It also appeared that the simulations of large systems would require longer simulated times in order to achieve equilibration, particularly near the melting temperature. By authors in [2] the choice of a $(5 \times 5 \times 5$ unit cells solid phase + liquid phase of an equivalent size) seems thus a good compromise in view of the accuracy of the results and the computing time.

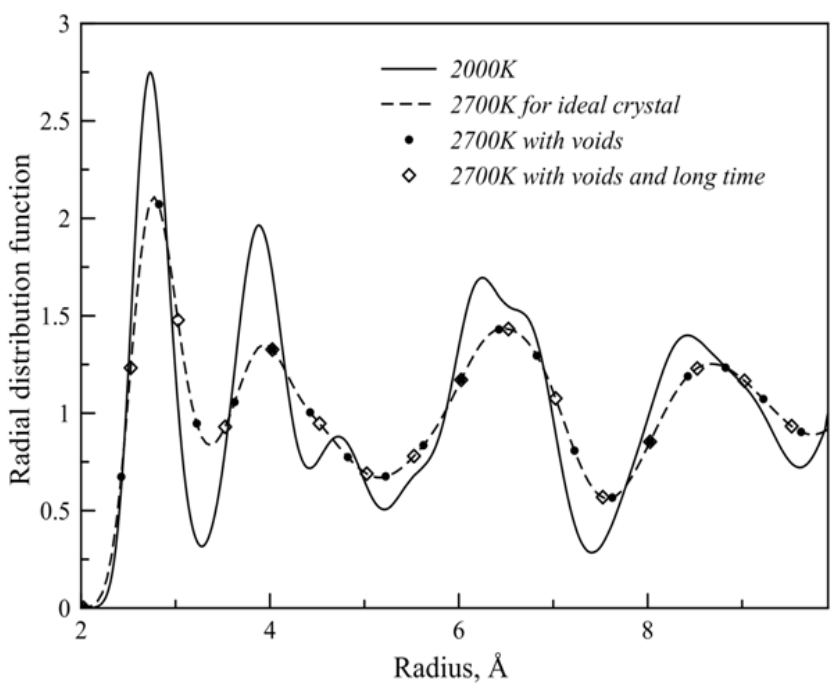

Figure 4. Radial distribution function (RDF) of oxygen atoms at the temperature of $2700 \mathrm{~K}$ and various simulation conditions: for the ideal crystal, for a crystal with voids, and with varying simulation period 100 ps and 10 ns. For comparison, RDF of oxygen at $2000 \mathrm{~K}$ is shown.

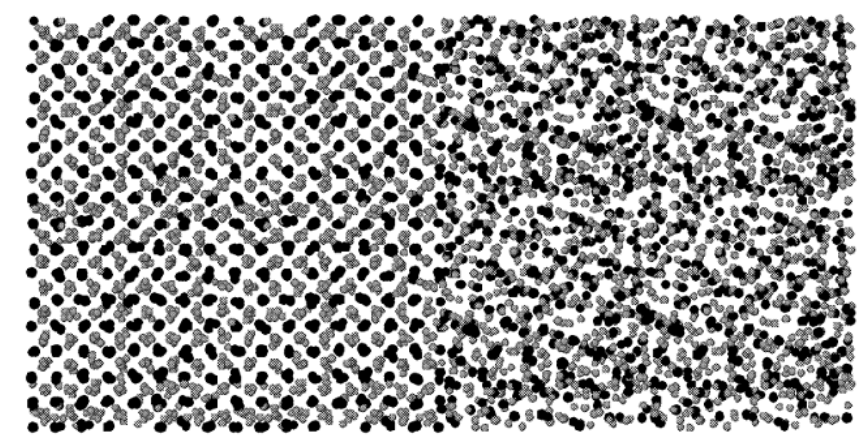

Figure 5.Initial configuration of the run for the determination of the melting temperature. Picture obtained in [2]. 
Table 2. The melting point of uranium dioxide for different initial temperatures and interatomic potentials are obtained in [2]. Bold data: coexistence of the solid and liquid phase; Italic data: solidification of the system; Normal data: liquefaction of the system.

\begin{tabular}{|c|c|c|c|c|c|}
\hline \multirow[t]{2}{*}{$\begin{array}{l}\text { Initial temperature, } \\
\qquad K\end{array}$} & Basak [8] & Karakasidis [2] & $\begin{array}{c}\text { Potential } \\
\text { Morelon [9] }\end{array}$ & Walker [2] & Yamada [10] \\
\hline & \multicolumn{5}{|c|}{ Temperature of melting point } \\
\hline 3000 & & $3100 \pm 50$ & & $3270 \pm 15$ & $3335 \pm 20$ \\
\hline 3050 & & $3145 \pm 50$ & $3340 \pm 25$ & & \\
\hline 3100 & & $3170 \pm 50$ & & & \\
\hline 3150 & & $3205 \pm 45$ & & & \\
\hline 3200 & $3525 \pm 25$ & $3075 \pm 25$ & $3510 \pm 20$ & $3360 \pm 40$ & $3525 \pm 20$ \\
\hline 3300 & $3610 \pm 25$ & & $3585 \pm 20$ & & \\
\hline 3400 & $3540 \pm 65$ & $3220 \pm 20$ & $3625 \pm 25$ & $3435 \pm 45$ & \\
\hline 3450 & $3540 \pm 65$ & & $3710 \pm 20$ & $3460 \pm 45$ & \\
\hline 3500 & $3545 \pm 60$ & & $3500 \pm 65$ & $3350 \pm 20$ & \\
\hline 3550 & $3440 \pm 15$ & & $3320 \pm 25$ & $\mathbf{3 5 3 0} \pm \mathbf{5 0}$ & \\
\hline 3600 & $3460 \pm 20$ & $3430 \pm 25$ & $3425 \pm 20$ & $3400 \pm 25$ & $3885 \pm 25$ \\
\hline 3800 & $3640 \pm 20$ & $3635 \pm 20$ & $3570 \pm 20$ & $3580 \pm 25$ & $4065 \pm 40$ \\
\hline 4000 & $3880 \pm 20$ & $3840 \pm 30$ & $3810 \pm 20$ & $3520 \pm 30$ & $4155 \pm 55$ \\
\hline
\end{tabular}

\section{Conclusion}

To determine phase transition temperatures more accurately, we suggested a method that uses the change rate of temperature dependence of the structure factor. In this case, the temperatures of melting and transition into the superionic state are $\sim 3100 \mathrm{~K}$ and $\sim 2600 \mathrm{~K}$, respectively. Theses temperatures much better conform to the experimental values of $3120 \mathrm{~K}$ and $2670 \mathrm{~K}$ than in the radial distribution function analysis method and other approaches. Other methods give the melting temperatures substantially higher $(3435-3600 \mathrm{~K})$ than the experimental value. Our method can be used for calculating transition temperatures in nanocrystals, and also in critical conditions, for example, under high pressure and/or under radiation when experimental measuring is difficult or impossible.

\section{Acknowledgements}

This work was supported by Russian Foundation for Basic Research (grant N 11-01-00311-a).

\section{References}

[1] K. Govers, S. Lemehov, M. Hou, M. Verwerft "Comparison of interatomic potentials for UO2. Part I: Static calculations" in Journal of Nuclear Materials, Vol. 366, 2007, pp. 161-177.

[2] K. Govers, S. Lemehov, M. Hou, M. Verwerft “Comparison of interatomic potentials for UO2 Part II: Molecular dynamics simulations" in Journal of Nuclear Materials, Vol. 376, 2008, pp.66-77.
[3] C. Kittel, Introduction to solid state physics. Wiley, New York, 1971.

[4] S. Yamasaki, T. Arima, K. Idemitsu "Evalution of Thermal Conductivity Hyperstoihiometric $\mathrm{UO}_{2+\mathrm{x}}$ by Molecular Dynamics Simulation" in International Journal of Thermophysics, Vol.28, №2, 2007, pp.661-673.

[5] Agrawal1 P.M., Rice B.M., Thompson D.L. "Molecular dynamics study of the effects of voids and pressure in defect-nucleated melting simulations" in J. Chem. Phys., Vol. 118,2003 , pp.9680-9684.

[6] J. Solca, A. J. Dyson, G. Steinebrunner, B. Kirchner and H. Huber "Melting curves for neon calculated from pure theory" in J. Chem. Phys., Vol. 108, 1998, pp.4107-4111.

[7] D. G. Gromov, S. A. Gavrilov "Manifestation of the heterogeneous mechanism upon melting of low-dimensional systems" in Physics of the Solid State, Vol. 51, N 10, 2009. pp 2135-2144.

[8] C.B. Basak, A.K. Sengupta, H.S. Kamath "Classical molecular dynamics simulation of $\mathrm{UO}_{2}$ to predict thermophysical properties" in J. Alloys and Comp., Vol. 360, 2003, pp. 210-216.

[9] N.-D. Morelon, D. Ghaleb "A new empirical potential for simulating the formation of defects and their mobility in uranium dioxide" in Phil. Mag., Vol. 83, 2003, pp. $1533-1550$.

[10] Yamada K., Kurosaki K., Uno M., Yamanaka S. Evaluation of thermal properties of uranium dioxide by molecular dynamics // J. Alloys and Comp. 2000. Vol. 307. P. 10-15.

[11] J.K. Fink "Thermophysical properties of uranium dioxide" in Journal of Nuclear Materials, Vol. 279, 2000, pp. 1-18. 